\title{
$\$$ Research Square \\ Household pesticide exposure and depression syndromes: evidence from NHANES, 2005-2014
}

Haiyan Chen

Guangzhou Center for Disease Control and Prevention

Guang Hao ( $\nabla$ haoguang2015@hotmail.com )

Jinan University https://orcid.org/0000-0003-3780-0223

Research article

Keywords: Exercise, household pesticide exposure, depression symptoms, general population

Posted Date: January 6th, 2020

DOI: https://doi.org/10.21203/rs.2.20077/v1

License: (c) (i) This work is licensed under a Creative Commons Attribution 4.0 International License.

Read Full License 


\section{Abstract}

\section{Background}

The effect of household pesticide exposure on depression symptoms in general population is underexplored, and the role of exercise in the association between pesticide exposure and depression symptoms is unclear. The goals of this study are to examine whether the association of household pesticide exposure and depression symptoms exist in general population, and, if so, whether exercise can attenuate the effect of household pesticide on depression symptoms.

Methods

We used data from the 2005-2014 NHANES (National Health and Nutrition Examination Surveys), including a total of 14708 US adult participants $\geq 20$ years old. Depression symptoms was assessed using the Patient Health Questionnaire (PHQ-9).

Results

The prevalence of depression symptoms was significantly higher in participants who exposed to household pesticide compared to those not $(28.9 \%$ vs. $21.1 \%, P<0.001)$. Household pesticide exposure was significantly associated with risk of depression symptoms. A significant interaction between exercise and pesticide exposure on depression symptoms was observed $(P=0.038)$. The prevalence of depression symptoms was significantly higher in pesticide exposure group compared to the control group (35.0\% vs. $24.9 \%, \mathrm{P}<0.001)$ in the population with light physical activity. However, we did not find a significant association in the group with moderate + vigorous physical activity.

\section{Conclusion}

This study further confirmed that household pesticide exposure is associated with a high risk of depression symptoms in the general population. More importantly, we for the first time reported that exercise tends to attenuate the effect of household pesticide exposure on depression symptoms.

\section{Background}

Pesticides are widely used in households to control insects. Household pesticide use in the United States of America continues to be very common, with use prevalence as high as $80-90 \%$ of households.[1] Previous findings have indicated that occupational or residential pesticide exposure is associated with an increased risk of many health problems, including asthma, diabetes, Parkinson's disease, birth and fetal defects, certain cancers, and psychiatric disorders.[2-4] Although the association between pesticide exposure and depression symptoms was found in several studies, most studies are limited in their generalization ability due to performing in occupational population, such as agricultural workers. $[2,5-7]$ Also, the controversial results exist. For example, Solomon and colleagues reported that past use of pesticides was not associated with current anxiety or depression symptoms in a cross-sectional study.[8] 
Household pesticide exposure may differ greatly from occupational exposure in terms of the frequency, duration, intensity, and type of exposure. However, the data on the association between household pesticide exposure and depression symptoms in the general population is scarce.

A Cochrane review concluded that exercise seems to improve depressive symptoms in people with a diagnosis of depression symptoms when compared with no treatment or control intervention, although highlighted that this should be interpreted with caution.[9] Similarly, another recent systematic review also reported that physical activity can confer protection against the emergence of depression symptoms regardless of age and geographical region.[10] Although the exact mechanisms which support the relationship are still poorly understood, previous studies suggested that the inflammatory response, physiological changes associated with exercise including cortisol, endorphin or monoamine levels, neurotransmitter function altering may play roles in it.[11] [12,13] In addition, previous studies have also indicated that physical activity may detox harmful chemicals from the body and can alleviate depression symptoms. $[14,15]$ Therefore, the goals of this study are to examine whether the associations of household pesticide exposure and depression symptoms exist in general population, and, if so, whether exercise can attenuate the effect of household pesticide on depression symptoms.

\section{Methods}

Study Population

NHANES (National Health and Nutrition Examination Surveys) is a series of large, multistage probability surveys designed to be representative of the US civilian, noninstitutionalized population that is conducted by the National Center for Health Statistics (NCHS) and Centers for Disease Control and Prevention. [16] 161616 Detailed descriptions of the survey design and data collection procedures are available elsewhere. [16] ${ }^{161616}$ The data are collected via an in-home interview and a visit to a mobile examination center. All participants provided written informed consent and the research ethics boards of the NCHS approved all protocols.

We used data from the 2005-2014 NHANES (including 5 cycles: 2005-2006, 2007-2008, 2009-2010, 2011-2012, and 2013-2014), and a total 14708 US adult participants $\geq 20$ years old met the following inclusion criteria were used in the study: 1) data available on age, sex, race, body mass index (BMI), recreational physical activity, education attainment, and poverty income ratio; 2 ) the participants responded to the questions on pesticide exposure and depression symptoms.

\section{Measures}

Exercise information in a typical week (yes/no) was self-reported during an interview. Moderate and vigorous recreational physical activity, where moderate recreational physical activity is defined as that activities cause a small increase in breathing or heart rate (such as brisk walking, bicycling, swimming, or volleyball for at least 10 minutes continuously); and vigorous recreational physical activity is defined as 
activities that causes large increases in breathing or heart rate like running or basketball for at least 10 minutes continuously (like running or basketball for at least 10 minutes continuously).

Household pesticide exposure was defined as those who responded to "In the past 7 days, were any chemical products used in home to control fleas, roaches, ants, termites, or other insects?"

Depression symptoms was assessed using the Patient Health Questionnaire (PHQ-9), which is a 9-item depression symptoms screening instrument that asks participants to choose 1 of 4 responses about the frequency of depressive during the previous 2 weeks.[17] Those scoring $\geq 10$ were considered as having moderate, moderately severe, or severe depression symptoms.

Antidepressant use was defined as taking at least one prescribed antidepressant medication in the past 30 days. We identified antidepressants using the Lexicon Plus ${ }^{\circledR}$ therapeutic classification (first-level category "Psychotherapeutic Agents", second level category "Antidepressants"), which included selective serotonin reuptake inhibitors, monoamine oxidase inhibitors, tricyclic antidepressants, serotoninnorepinephrine reuptake inhibitors, phenylpiperazine, and miscellaneous antidepressants.[18]

For psychological counseling, we defined counseling and various types of therapy as treatment with a mental health professional, which was measured by the survey question, "During the past 12 months, have you seen or talked to a mental health professional such as a psychologist, psychiatrist, psychiatric nurse, or clinical social worker about your health?"

We defined depression symptoms as PHQ-9 total scores $\geq 10$, or taking at least one prescribed antidepressant medication in the past 30 days, or receiving psychological counseling treatment.

Weight and height were measured by trained technicians in mobile examination centers who used standardized procedures. Race was based on proxy- or self-report and was categorized as non-Hispanic white, non-Hispanic black, Mexican-American, or other categories. The poverty income ratio is the ratio of a family's income to the US Census Bureau's poverty threshold, which is adjusted for family size and is updated annually for inflation. The poverty income ratio was used as the indicator of socioeconomic status in the analyses. Participants were categorized as never smokers (individuals who have smoked < 100 cigarettes in life), former smokers (having smoked $>100$ cigarettes in life but do not currently smoke), and current smokers.

Statistical Analysis

All variables were checked for normality of distribution, and appropriate transformations were applied when necessary. Continuous variables are presented as mean $\pm S D$, whereas categorical variables are presented as cases (n). Logistic regression was used to calculate the odds for the association of pesticide exposure with depression symptoms, and the NHANES sampling weights and complex survey design were considered in the analysis. Univariate analyses were performed in Model 1. Model 2 adjusted for age, sex, and race; Model 3 more adjusted for BMI, recreational physically inactive, marital status, education attainment, and poverty income ratio. In addition, the interactions between pesticide exposure 
and recreational physical activity. The NHANES does not provide details on antidepressant use and psychological counseling, but the individuals seeking psychological counseling might suffer from other mental illnesses rather than depression, and antidepressants were prescribed for reasons other than depression, so we performed a sensitivity analysis by excluding the participants with antidepressant medication or receiving psychological counseling treatment (1608 participants). In addition, we performed another sensitivity analysis using the PHQ-9 score as a continuous variable. All data analyses were performed using Stata software version 12.1 (STATA Corp., TX, US). A two-sided $P<0.05$ was considered statistically significant.

\section{Results}

There were 14708 participants eligible for our final analysis, of them $1835(12.5 \%)$ participants were selfreported an exposure of household pesticide. The mean age of participants was $48.0 \pm 17.7$ years, $55.6 \%$ of the participants were female, and $22.1 \%$ participants with depression syndromes. The characteristics of the pesticide exposure and control group were statistically significantly different except for sex (Table 1).

The prevalence of depression symptoms was significantly higher in participants who exposed to household pesticide compared to those not $(28.9 \%$ vs. $21.1 \%, \mathrm{P}<0.001)$. Table 2 shows the relationship between household pesticide exposure and risk of depression, in which participants who exposed to household pesticide had a higher odds ratio ([OR]; OR = 1.46, 95\% Cl[confidence intervals]: 1.24-1.72) for depression (model 1). Additionally, adjusted for age, sex, and race (model 2), the OR remains the same; and further adjusted for BMI, recreational physically inactive, marital status, education attainment, and income ratio (model 3), the OR was 1.32 (95\% Cl: 1.12-1.56) (Supplementary STable 1).

A significant interaction between physical activity and household pesticide exposure on depression is observed $(P=0.038)$ (Fig. 1).

Stratified analysis was performed according to physical activity (moderate + vigorous vs. light). In the group with moderate + vigorous physical activity, the prevalence of depression symptoms between household pesticide-exposed and the non-exposed group is not statistically different ( $21.0 \%$ vs. $17.2 \%, P$ $=0.305$ ). After adjusting for other covariates, the association between household pesticide exposure and depression symptoms is not statistically significant $(\mathrm{OR}=1.12,95 \% \mathrm{Cl}: 0.90-1.41)$. However, household pesticide exposure is significantly associated with depression symptoms $(\mathrm{OR}=1.50,95 \% \mathrm{Cl}$ : $1.20-1.86)$ in the population with light physical activity. The prevalence of depression symptoms is significantly higher in household pesticide exposure group compared to the control group $(35.0 \%$ vs. $24.9 \%, \mathrm{P}<0.001)$ (Table 3). Sensitivity analysis showed similar results (Supplementary STable 2-5, and SFigure 1).

\section{Discussion}

We found that household pesticide exposure is significantly associated with depression symptoms in the general population. We for the first time reported that there is an interaction between exercise with 
pesticide exposure for the risk of depression symptoms, indicating that exercise tends to attenuate the effect of pesticide exposure on depression symptoms.

Previous studies have reported that pesticide exposure has been associated with depressive disorders in occupational settings. $[2,19]$ For example, after an average of 12.1 years follow-up, Beard et al. reported a positive association between the use of pesticides and depression among male private pesticide applicators the Agricultural Health Study.[19] Another cohort study, the Colorado Farm Family Health, and Hazard Surveillance (CFFHHS) project, also showed that a history of pesticide poisoning was associated with a high risk of depression.[20] A systematic review study published in 2013 including 11 studies on pesticide exposure and depression found that depression or other psychiatric disorders have shown increased risks associated with previous pesticide poisoning in 5 studies, with statistically significant OR ranging from 2.08 to 5.95 , indicating that scientific evidence of association between pesticide exposure and depression is still very limited and inconclusive.[2] An ecological study by Parrón et al. demonstrated that the population living in areas with high pesticide use had an increased risk for Alzheimer's disease and suicide attempts and that males living in these areas had increased risks for polyneuropathies, affective disorders and suicide attempts at the level of the general population.[21] This study support and extends previous findings and reported that pesticide exposure is associated with depressive disorders in the general population. However, the underlying mechanisms which support this relationship are still poorly understood. Previous studies have shown that many pesticides have neurotransmitter toxicity, including inhibition of cholinesterase activity, increasing excitatory amino acid, decreasing the level of Dopamine,[22] which could be the possible mechanisms in the association between pesticide exposure and depressive disorders.

To date, numerous studies, including randomized controlled trials, prospective study, and meta-analysis, have demonstrated the benefits of physical activity on depression.[10, 23, 24] In our study, an interaction between physical activity and pesticide exposure in term of depression is observed, but still, the underlying mechanisms are unclear. One possible explanation is that physical activity may promote the clearance of harmful chemicals with neurotoxicity from the body. An animal study demonstrated that cardiovascular exercise training activates PGC-1a1:PPARa/ $\delta: K A T$, which controls plasma and brain kynurenine/kynurenic acid balance and has a protective effect on stress-induced depression in the mouse.[15] This study suggesting that cardiovascular exercise may detox harmful chemicals from the body and then can alleviate stress-induced depression. Another study including 133 students by Zaitseva et al. found that increased physical activity is associated with decreased hair copper, vanadium, bismuth, and mercury content in comparison to the low physical activity groups.[14] Other biochemical are also likely responsible, including biological mechanisms through which exercise increases neurogenesis and reduces inflammatory and oxidant markers and activates the endocannabinoid system.[10, 25, 26]

There is unclarity about the chemical types, intensity, and forms of exposure in relation to the risk of depression.[3] A study of 567 agricultural workers in France evaluated the effect of several pesticide families, reported positive associations between depression and exposure to herbicides in general and dinitrophenol herbicides, but not exposure to any pesticide, fungicides, insecticides, or the other 12 
herbicide families.[27] In the Agricultural Health Study, depression was positively associated in each case group with ever-use of two pesticide classes, fumigants and organochlorine insecticides, as well as with ever-use of seven individual pesticides: the fumigants aluminum phosphide and ethylene dibromide; the phenoxy herbicide 2,4,5-T; the organochlorine insecticide dieldrin; and the OPs diazinon, malathion, and parathion.[19] Therefore, more research is needed to identify the specific harmful components, toxicity, and dose of pesticides for depression.

\section{Limitations and strengths}

Strengths of our study include the large sample size and performed in the general population. In addition, we for the first time reported the interaction between physical activity and household pesticide exposure on risk of depression. There were also several limitations in our study. One limitation is that the crosssectional nature of the present study precludes the inference of the cause-effect relationship. Another limitation is that the NHANES study did not collect more detailed information on household pesticide, such as chemical types and intensity, which may have a varied effect on depression. The third one is the information on pesticide use and exercise was self-reported and could be misclassified. Fourth, NHANES has geographical limitations and may not be sufficient to represent other geographic regions. Last not the least, although the PHQ-9 has excellent reliability and validity in primary care, it useful only for screening purposes for "current major depressive episode" as a result of its low positive predictive value.[28] Therefore, we performed a sensitivity analysis using the PHQ-9 score as a continuous variable, and found similar results.

\section{Conclusion}

In conclusion, this study adds further evidence that household pesticide exposure is associated with a high risk of depression in the general population. We for the first time reported that there was an interaction between exercise and household pesticide exposure on the depression indicating that moderate or vigorous physical activity tend to attenuate the effect of household pesticide exposure on depression. Our results highlight the importance of the cautious use of household pesticide because the chronic effects of poisoning may contribute to a high risk of depression. Further cohort studies will be necessary to confirm our results and to identify the specific harmful components, toxicity, and a dose of pesticide for depression and other neuropsychological disorders.

\section{Abbreviations}

BMI: body mass index; Cl: Confidence intervals; PHQ: Patient Health Questionnaire; OR: Odds ratio; NHANES: National Health and Nutrition Examination Surveys

\section{Declarations}

Acknowledgments 
The authors thank the NHANES participants and the staff numbers for their contributions of the data and data collection.

\section{Authors' contributions}

Haiyan Chen contributed to the performance of the statistical analysis and interpretation of the results and helped in writing the first draft of this manuscript. Guang Hao contributed to the conceptualization of the analysis and interpretation of the results and wrote the first draft of this manuscript and subsequent revisions.

\section{Funding}

This study was partly supported by "the Fundamental Research Funds for the Central Universities (21619332)".

\section{Availability of data and materials}

The data of this study are available at https://www.cdc.gov/nchs/nhanes/index.htm.

\section{Ethics approval and consent to participate}

All participants provided written informed consent and the research ethics boards of the National Center for Health Statistics approved all protocols.

\section{Consent for publication}

Not applicable.

\section{Competing interests}

All authors declare no conflict of interest.

\section{References}

1. Narayan S, Liew Z, Paul K, Lee P-C, Sinsheimer JS, Bronstein JM, Ritz B: Household organophosphorus pesticide use and Parkinson's disease. International journal of epidemiology 2013, 42(5):1476-1485.

2. Freire $C$, Koifman S: Pesticides, depression and suicide: a systematic review of the epidemiological evidence. International journal of hygiene and environmental health 2013, 216(4):445-460.

3. Koh SB, Kim TH, Min S, Lee K, Kang DR, Choi JR: Exposure to pesticide as a risk factor for depression: A population-based longitudinal study in Korea. Neurotoxicology 2017, 62:181-185.

4. Kim KH, Kabir E, Jahan SA: Exposure to pesticides and the associated human health effects. The Science of the total environment 2017, 575:525-535. 
5. Beard JD, Hoppin JA, Richards M, Alavanja MC, Blair A, Sandler DP, Kamel F: Pesticide exposure and self-reported incident depression among wives in the Agricultural Health Study. Environmental research 2013, 126:31-42.

6. Onwuameze OE, Paradiso S, Peek-Asa C, Donham KJ, Rautiainen RH: Modifiable risk factors for depressed mood among farmers. Annals of clinical psychiatry : official journal of the American Academy of Clinical Psychiatrists 2013, 25(2):83-90.

7. Tarone RE, Alavanja MC, Zahm SH, Lubin JH, Sandler DP, McMaster SB, Rothman N, Blair A: The Agricultural Health Study: factors affecting completion and return of self-administered questionnaires in a large prospective cohort study of pesticide applicators. American journal of industrial medicine 1997, 31(2):233-242.

8. Solomon C, Poole J, Palmer KT, Peveler R, Coggon D: Neuropsychiatric symptoms in past users of sheep dip and other pesticides. Occupational and environmental medicine 2007, 64(4):259-266.

9. Rimer J, Dwan K, Lawlor DA, Greig CA, McMurdo M, Morley W, Mead GE: Exercise for depression. The Cochrane database of systematic reviews 2012(7):CD004366.

10. Schuch FB, Vancampfort D, Firth J, Rosenbaum S, Ward PB, Silva ES, Hallgren M, Ponce De Leon A, Dunn AL, Deslandes AC et al: Physical Activity and Incident Depression: A Meta-Analysis of Prospective Cohort Studies. The American journal of psychiatry 2018, 175(7):631-648.

11. Hamer M, Endrighi R, Poole L: Physical activity, stress reduction, and mood: insight into immunological mechanisms. Methods in molecular biology 2012, 934:89-102.

12. Lucassen PJ, Meerlo P, Naylor AS, van Dam AM, Dayer AG, Fuchs E, Oomen CA, Czeh B: Regulation of adult neurogenesis by stress, sleep disruption, exercise and inflammation: Implications for depression and antidepressant action. European neuropsychopharmacology : the journal of the European College of Neuropsychopharmacology 2010, 20(1):1-17.

13. Duclos M, Gouarne C, Bonnemaison D: Acute and chronic effects of exercise on tissue sensitivity to glucocorticoids. Journal of applied physiology 2003, 94(3):869-875.

14. Zaitseva IP, Skalny AA, Tinkov AA, Berezkina ES, Grabeklis AR, Skalny AV: The influence of physical activity on hair toxic and essential trace element content in male and female students. Biological trace element research 2015, 163(1-2):58-66.

15. Agudelo LZ, Femenia T, Orhan F, Porsmyr-Palmertz M, Goiny M, Martinez-Redondo V, Correia JC, Izadi M, Bhat M, Schuppe-Koistinen I et al: Skeletal muscle PGC-1alpha1 modulates kynurenine metabolism and mediates resilience to stress-induced depression. Cel/ 2014, 159(1):33-45.

16. Centers for Disease Control and Prevention. https://www.cdc.gov/nchs/nhanes/index.htm. Accessed May 1, 2018.

17. Kroenke K, Spitzer RL, Williams JB: The PHQ-9: validity of a brief depression severity measure. Journal of general internal medicine 2001, 16(9):606-613.

18. Wang Y, Lopez JM, Bolge SC, Zhu VJ, Stang PE: Depression among people with type 2 diabetes mellitus, US National Health and Nutrition Examination Survey (NHANES), 2005-2012. BMC psychiatry 2016, 16:88. 
19. Beard JD, Umbach DM, Hoppin JA, Richards M, Alavanja MC, Blair A, Sandler DP, Kamel F: Pesticide exposure and depression among male private pesticide applicators in the agricultural health study. Environmental health perspectives 2014, 122(9):984-991.

20. Beseler CL, Stallones L: A cohort study of pesticide poisoning and depression in Colorado farm residents. Annals of epidemiology 2008, 18(10):768-774.

21. Parron T, Requena M, Hernandez AF, Alarcon R: Association between environmental exposure to pesticides and neurodegenerative diseases. Toxicology and applied pharmacology 2011, 256(3):379385.

22. Liu L: Toxicity of Pesticide on Neurotransmitter. Advanced Materials Research 2012, 518-523:20452048.

23. Dunn AL, Trivedi MH, Kampert JB, Clark CG, Chambliss HO: Exercise treatment for depression: efficacy and dose response. American journal of preventive medicine 2005, 28(1):1-8.

24. Conn VS: Depressive symptom outcomes of physical activity interventions: meta-analysis findings. Annals of behavioral medicine : a publication of the Society of Behavioral Medicine 2010, 39(2):128138.

25. Schuch FB, Deslandes AC, Stubbs B, Gosmann NP, Silva CT, Fleck MP: Neurobiological effects of exercise on major depressive disorder: A systematic review. Neuroscience and biobehavioral reviews 2016, 61:1-11.

26. Brellenthin AG, Crombie KM, Hillard CJ, Koltyn KF: Endocannabinoid and Mood Responses to Exercise in Adults with Varying Activity Levels. Medicine and science in sports and exercise 2017, 49(8):1688-1696.

27. Weisskopf MG, Moisan F, Tzourio C, Rathouz PJ, Elbaz A: Pesticide exposure and depression among agricultural workers in France. American journal of epidemiology 2013, 178(7):1051-1058.

28. Inoue T, Tanaka T, Nakagawa S, Nakato Y, Kameyama R, Boku S, Toda H, Kurita T, Koyama T: Utility and limitations of PHQ-9 in a clinic specializing in psychiatric care. BMC psychiatry 2012, 12:73.

\section{Tables}


Table 1. Characteristics of the study sample

\begin{tabular}{llll}
\hline Variable & Pesticide exposure $(\mathrm{n}=1835)$ & $\begin{array}{l}\text { Control } \\
(\mathrm{n}=12873)\end{array}$ & $P$ value \\
\hline $\begin{array}{l}\text { Age (years) } \\
\text { Sex (Females, \%) }\end{array}$ & $49.9 \pm 17.3$ & $47.8 \pm 17.8$ & $<0.001$ \\
\hline Race (\%) & $1009(55.0)$ & $7163(55.6)$ & 0.596 \\
\hline Non-Hispanic white & $752(41.0)$ & $6393(49.7)$ & $<0.001$ \\
\hline Non-Hispanic black & $514(28.0)$ & $2487(19.3)$ & \\
\hline Mexican-American & $277(15.1)$ & $1851(14.4)$ & \\
\hline Other & $292(15.9)$ & $2142(16.6)$ & \\
\hline Body mass index $\left(\mathrm{kg} / \mathrm{m}^{2}\right)$ & $29.8 \pm 7.5$ & $29.4 \pm 7.2$ & 0.008 \\
\hline Smoking status $(\%)$ & & & \\
\hline Current smoker & $869(47.4)$ & $6769(52.6)$ & $<0.001$ \\
\hline Former smoker & $405(22.1)$ & $2874(22.3)$ & \\
\hline Never smoker & $561(30.6)$ & $3230(25.1)$ & \\
\hline Marital status $(\%)$ & $822(44.8)$ & $6420(49.9)$ & $<0.001$ \\
\hline Education attainment & & & \\
\hline$<9 \quad$ years & $201(11.0)$ & $1116(8.7)$ & $<0.001$ \\
\hline 9-11 years & $341(18.6)$ & $1982(15.4)$ & \\
\hline 12 years & $450(24.5)$ & $3021(23.5)$ & \\
\hline$>12$ years & $843(45.9)$ & $6757(52.5)$ & \\
\hline Vigorous/ Moderate RPA(\%) & $805(43.9)$ & $2.5 \pm 1.6$ & $<0.001$ \\
\hline Poverty income ratio & $2.1 \pm 1.5$ & $2715(21.1)$ & $<0.001$ \\
\hline Depression symptoms $(\%)$ & $530(28.9)$ & &
\end{tabular}

$\mathrm{PRA}=$ recreational physically inactive 
Table 2. The associations between pesticide use in home and depression symptoms

OR $\quad 95 \%$ CI $\quad P$ value

\section{Model 1}

Control

Reference

Pesticide exposure

1.46

$1.24-1.72$

$<0.001$

Model 2

Control

Reference

Pesticide exposure

1.46

$1.24-1.72$

$<0.001$

Model 3

Control

Reference

Pesticide exposure

1.32

$1.12-1.56$

0.001

Model 1 unadjusted

Model 2 adjusted for age, sex, and race.

Model 3 adjusted for age, sex, race, body mass index, recreational physically inactive, marital status, education attainment, and poverty income ratio.

Table 3. The associations between pesticide use in home and depression symptoms by physical activity

$\begin{array}{lll}\text { OR } & 95 \% \mathrm{CI} & P \text { value }\end{array}$

\section{Light}

Control

Reference

Pesticide exposure

1.50

$1.20-1.86$

$<0.001$

Vigorous/ Moderate

Control

Reference

Pesticide exposure

1.12

$0.90-1.41$

0.305

Adjusted for age, sex, race, body mass index, marital status, education attainment, and poverty income ratio.

\section{Figures}




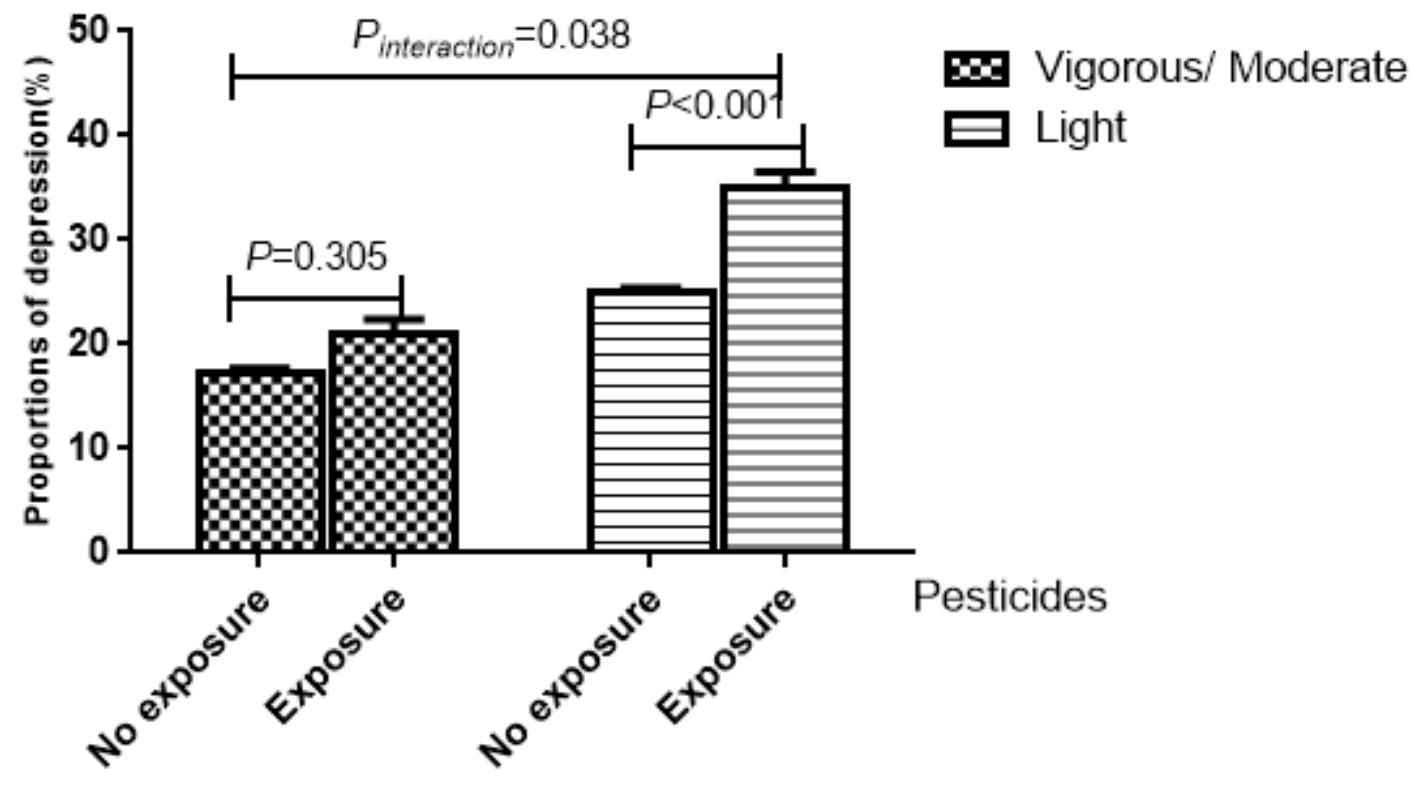

Figure 1

The proportions of depression symptoms in pesticide in pesticide-exposed and non-exposed group by physical activity.

\section{Supplementary Files}

This is a list of supplementary files associated with this preprint. Click to download.

- supplementary.docx 